\title{
Obligatorische Messung der Patientenzufriedenheit
}

\section{Umpitz}

Dr. med., Schweizerische Zentralstelle zur Qualitätsförderung im Gesundheitswesen (SZQG $\left.{ }^{1}\right)$

\section{Geschichtliche Entwicklung}

Die ursprüngliche Idee, Patienten systematisch nach ihrer Zufriedenheit zu befragen, hatte der Berner Chirurg Theodor Kocher (1841-1917), bekannt durch seine Arbeiten zur Pathologie und Chirurgie der Schilddrüse. Operationsbedingte Schädigungen des Nervus recurrens erschwerten jedoch die Durchführung der Befragungen und sorgten für eine schlechte Rücklaufquote. Da Kocher von den anfänglichen Schwierigkeiten entmutigt und der anstehenden Verleihung des Nobelpreises abgelenkt war, geriet das Vorhaben von Zufriedenheitsbefragungen anschliessend in Vergessenheit. Es sollten fast 100 Jahre vergehen, bis Medizinhistoriker die «Kocher'sche Idee» wiederentdeckten und die Grundlage für die Entwicklung standardisierter Messinstrumente im vergangenen Jahrzehnt schufen.
1 Die SZQG berät die FMH in Fragen zu evidenzbasierter Medizin und Versorgungsqualität. 2 Patienten-Information zur Zufriedenheit mit Arztpraxen in Zürich.

\section{Schwächen heutiger Zufriedenheitsmessungen}

Patientenzufriedenheit ist als Teilaspekt der ambulanten Versorgungsqualität zu betrachten [1]. Es gibt von ihr jedoch kein einheitliches Konstrukt - was die Vielzahl von Befragungsinstrumenten erklärt, die sich substantiell voneinander unterscheiden und Vergleiche zwischen Praxen unmöglich machen [2]. Neben Fragebögen aus den Nachbarländern haben in der Schweiz auch das Oltener Patientenzufriedenheitsinventar (OPI) und PIzZA-Zürich ${ }^{2}$ eine gewisse Bedeutung erlangt. Schwächen nahezu aller Befragungsinstrumente sind ihre schlechte Lesbarkeit, die Unverständlichkeit der Fragen und der hohe Zeitaufwand zum Ausfüllen der Fragebögen. Hinzu kommt, dass Reliabilität, inhaltliche Validität und Übertragbarkeit oftmals nicht gezeigt worden sind. Nicht zuletzt besteht das grundsätzliche Problem aller zurzeit verwendeten Messinstrumente in der fehlenden Kopplung der Patientenzufriedenheit an externe Qualitätskriterien; daher liessen sich über die alleinige Erfassung der Patientenzufriedenheit bisher keine Rückschlüsse auf die objektive Qualität ambulanter Versorgung ziehen.

\section{DUPS-Q: ein Quantensprung in der Patientenbefragung}

Die European Foundation for Quality Management beauftragte 2009 eine Arbeitsgruppe der Universität Leiden (NL) mit der Entwicklung und Validierung eines praxistauglichen Fragebogens. Die Arbeiten wurden im vergangenen Jahr fertiggestellt und ihre Publikation erregte weltweites Aufsehen [3]. Der neue Fragebogen «Dutch Patient Satisfaction Questionnaire» (DUPS-Q) erfasst nur zwei Wahrnehmungsdimensionen (Zufriedenheit mit der Versorgung und der Praxisführung) und kommt mit 16 leicht verständlichen Fragen aus trotzdem wird ein breites Spektrum an Erlebnisinhalten abgedeckt (s. Abb. 1).

Ausgedehnte Validierungsstudien haben die zuverlässige und valide Messung der Patientenzufriedenheit
Lassen auch Sie den DUPS-Q-Fragebogen von Ihren Patienten ausfüllen und erfahren Sie so, wie zufrieden Ihre Patienten mit Ihrer Performance sind. 
Praxisstempel

FRAGEBOGEN ZUR PATIENTENZUFRIEDENHEIT

Patientenangaben

Vor- und Nachname

Geburtsdatum

Datum des Arztbesuchs

Liebe Patientin, lieber Patient

Mit diesem Fragebogen möchten wir von Ihnen erfahren, wie zufrieden Sie mit der Arztpraxis und ihrer medizinischen Versorgung sind - bezogen auf den heutigen Besuch. Ihre Antworten helfen mit, zukünftig eine bessere Versorgung zu niedrigeren Kosten anbieten zu können. Die Befragung ist anonym, das heisst, Sie wissen nicht, wer ausser Ihrer Ärztin / Ihrem Arzt und Ihrer Krankenkasse Einblick in Ihre Angaben erhält. Vielen Dank für Ihre Unterstützung!

Wie zufrieden sind Sie mit ...

Sehr unzufrieden

Eher

Eher unzufrieden zufrieden

Sehr

den erhaltenen Informationen

$\begin{array}{ll}\square & \square \\ \square & \square \\ \square & \square \\ \square & \square \\ \square & \square \\ \square & \square \\ \square & \square \\ \square & \square\end{array}$

der Wartezeit in der Praxis

der Praxisausstattung

dem Zeitschriftenangebot

dem Umgangston in der Praxis

der Sauberkeit des Personals

der Gründlichkeit Ihrer Ärztin / Ihres Arztes

der Kompetenz Ihrer Ärztin / Ihres Arztes

der Bereitschaft zur Überweisung an eine

kompetente Ärztin / einen kompetenten Arzt

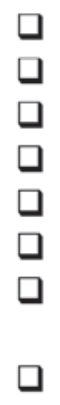

dem heutigen Arztbesuch insgesamt

Ich werde die Ärztin / den Arzt weiterempfehlen

$\begin{array}{ll}\square & \square \\ \square & \square \\ \square & \square \\ \square & \square \\ \square & \square \\ \square & \square \\ \square & \square \\ \square & \square\end{array}$




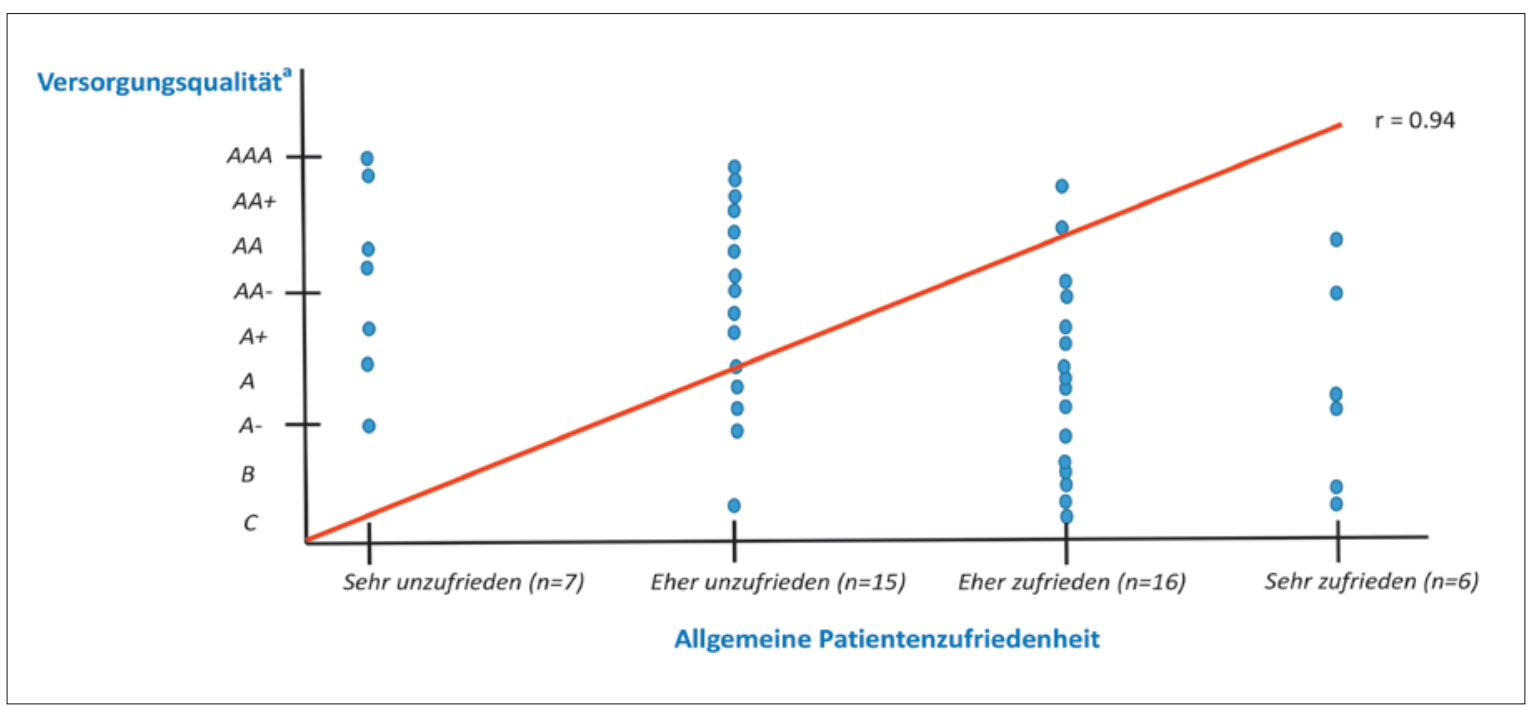

Abbildung 2: Auswertung der Zufriedenheit von 44 Zürcher Patienten - erhoben mit dem DUPS-Q. Es besteht eine sehr hohe Korrelation zwischen dem Ausmass der allgemeinen Patientenzufriedenheit und der individuellen Versorgungsqualität ( ${ }^{\text {e }}$ ermittelt nach Fitch Ratings).

bestätigt. Völlig unerwartet war die Erkenntnis, dass auf individueller Betrachtungsebene die Zufriedenheitswerte sehr stark mit der jeweils objektiv beurteilten Versorgungsqualität korrelieren [4]. Somit lassen sich unmittelbar Rückschlüsse auf die Qualität des Patientenmanagements und den Behandlungserfolg ziehen. Dass dies auch für Schweizer Patienten zutrifft, zeigte eine vor kurzem abgeschlossene Studie an $44 \mathrm{~Pa}$ tienten aus Zürcher Arztpraxen (Publikation in Vorbereitung; s. Abb. 2).

\section{Implementierungsplan für die Schweiz}

In der EU ist eine Verordnung in Vorbereitung, die den Einsatz von DUPS-Q im ambulanten Sektor verpflichtend vorschreiben wird. Auch für die Schweiz bestehen bereits konkrete Pläne für die Implementierung. Ein weiterer Vorteil: Der Fragebogen ist bereits für die Landessprachen Deutsch, Französisch und Italienisch validiert.

Die Implementierung in der Schweiz soll im Rahmen eines Drei-Schritte-Programms erfolgen: 1. Übergangsphase bis März 2018. Ärztinnen und Ärzte sind allerdings gut beraten, den Fragebogen bereits im kommenden Jahr in den Praxisbetrieb zu integrieren und Routine bei der Abgabe und beim Einsammeln zu entwickeln. 2. Ab dem 1. April 2018 wird das Ausfüllen des Fragebogens zur Pflicht - und zwar für jeden Arztbesuch. Die ausgefüllten Originale werden mit der Abrechnung an die Krankenkassen für gezielte Auswertungen weitergeleitet. Patienten, die den Fragebogen nicht ausfüllen, müssen einen höheren Selbstbehalt leisten. 3. Für die Zeit ab dem 1. April 2020 bestehen bereits
Überlegungen in Richtung konkreter qualitätssichernder Massnahmen. So sollen systematisch auftretende Zufriedenheitsdefizite in einer Praxis in gezielte Fortbildungsmassnahmen einmünden (z.B. für bestimmte Erkrankungen oder bezüglich der Praxisführung). Kontrovers wird noch die Einführung eines «Zufriedenheitsfaktors» für den Abrechnungsbetrag diskutiert, d.h. eine Kostenkorrektur durch das Ausmass an Patientenzufriedenheit. Es bestehen aber kaum Zweifel, dass sich dieses «Pay-for-performance-Modell» langfristig durchsetzen wird.

Als Leserin und Leser der Schweizerischen Ärztezeitung können Sie bereits heute DUPS-Q-Fragebögen bestellen - wahlweise 100, 200 oder 500 Exemplare in der gewünschten Landessprache (www.emh.ch/dups-qFragebogen).

\section{Bildnachweise}

Foto: @ Prykhodov | Dreamstime.com, bearbeitet von EMH Fragebogen: Schweizerische Zentralstelle zur Qualitätsförderung im Gesundheitswesen (SZQG)

Diagramm: M. Umpitz

\section{Literatur}

1 Ryan M, Scott DA, Reeves C et al. Eliciting public preferences for healthcare: a systematic review of techniques. Health Technol Assess. 2001;5:1-186.

2 Berger B, Lenz M, Mühlhauser I. Patient zufrieden, Arzt gut? Inwiefern ist Patientenzufriedenheit ein Indikator für die Qualität der hausärztlichen Versorgung? Eine systematische Übersichtsarbeit. Z Evid Fortbild Qual Gesundheitswesen. 2008;102:299-306.

3 Bouter H, Niekerk A, Van Veeteren S. DUPS-Q: Nu zitten wij met de gebakken peren. Nederl Tijdschr Management Gezondheitszorg. 2016;55:164-78.

4 Niekerk A, Van Veeteren S, Bouter H. Wie boter op zijn hoofd heeft moet niet in de zon lopen (DUPS-Q). Ned J Kwaliteit Med Gezond. 2016;12:81-9. 